\title{
PROPERTIES OF A VORTEX STREET OF FINITE VORTICES*
}

\author{
P. G. SAFFMAN $\dagger$ AND J. C. SCHATZMAN $\dagger$
}

\begin{abstract}
Steady solutions of the Euler equations are calculated for an infinite array of vortices, consisting of two staggered parallel rows of identical vortices of finite area and uniform vorticity. These models are similar to the "vortex streets" studied theoretically by von Kármán and others, except that here vortices of finite rather than infinitesimal area are employed.
\end{abstract}

Key words. Kármán vortex street, laminar wake

1. Introduction. For a certain range of Reynolds number, a regular pattern of vortices is observed in the wake of a two-dimensional blunt body placed in a uniform stream. In his classic work, von Kármán modeled the problem with an infinite street of point vortices (see Kármán [4], [5], Kármán and Rubach [6]). This approximate approach has several limitations, among which are the infinite kinetic energy and difficulty in fitting the model to flow past a body.

To improve the model, the vortices are herein allowed to be of finite area, but uniform vorticity. An integrodifferential equation is then solved to obtain the steady shapes of the vortices. This paper is a report of the first part of a study of the wake flow problem, and describes only properties of the steady solutions for the infinite vortex array. Subsequent papers will report on a stability analysis of the steady states, and on relating this model to the wake flow problem.

2. Formulation. Consider an infinite array of uniform two-dimensional vortices, consisting of one row of identical vortices of area $A$ and strength $-\Gamma$ with centroids at positions $x=0, \pm l, \pm 2 l, \pm 3 l, \cdots, y=0$, and of a second row of identical vortices of area $A$ and strength $+\Gamma$ with centroids at $x=d, d \pm l, d \pm 2 l, d \pm 3 l, \cdots, y=-h$. Let there be superimposed a uniform flow $U_{s}$ in the $x$ direction, at infinity, as in Fig. 1. It is assumed

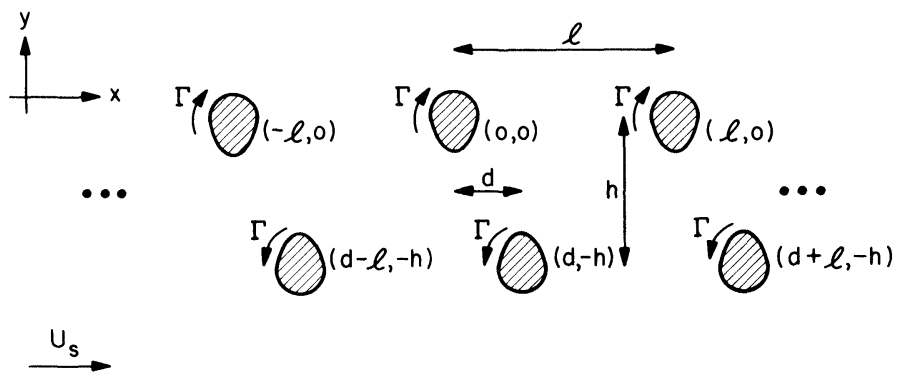

FIG. 1. The configuration of the fully infinite vortex street with arbitrary stagger.

that the flow is inviscid, incompressible, two-dimensional and, outside the vortices, irrotational. This paper deals with steady flows of this kind, principally with $d / l=0.5$ (for values other than 0 and 0.5 and for the symmetric periodic cases considered here, the street does not move parallel to itself; see Rosenhead [9]).

* Received by the editors September 10, 1980, and in revised form February 18, 1981.

$\uparrow$ Applied Mathematics Department, California Institute of Technology, Pasadena, California 91125. This work was supported by the U.S. Department of Energy (Office of Basic Energy Sciences). 
The complex potential outside the vortices can be written (with obvious notation)

$$
w(z)=\frac{i \Gamma}{2 \pi A}\left\{\iint_{\Sigma_{1}} \log \sin \frac{\pi}{l}\left(z-z^{\prime}\right) d x^{\prime} d y^{\prime}-\iint_{\Sigma_{2}} \log \sin \frac{\pi}{l}\left(z-z^{\prime}\right) d x^{\prime} d y^{\prime}\right\}+U_{s} z
$$

where $\Sigma_{1}, \Sigma_{2}$ refer to the cross-sections of one vortex in the upper and lower rows, respectively. By applying a Green's theorem, the complex velocity can be written as a line integral around the boundaries of the vortices:

$$
u+i v=\frac{\Gamma}{2 \pi A}\left\{\int_{\Sigma_{1}} \log \left|\sin \frac{\pi}{l}\left(z-z^{\prime}\right)\right| d z^{\prime}-\int_{\Sigma_{2}} \log \left|\sin \frac{\pi}{l}\left(z-z^{\prime}\right)\right| d z^{\prime}\right\}+U_{s} .
$$

The requirement that the velocity field be tangent to the boundary of the vortices then determines the steady shapes of the vortices, apart from the scaling, as a function of the three dimensionless parameters $d / l \equiv \mu, h / l \equiv \kappa$, and $A / l^{2} \equiv \alpha$.

To simplify the calculations, the vortices in the two rows are assumed to have identical shapes, differing only in position and orientation. There are two reasonable choices of symmetry: invariance to reflection about the line $y=-h / 2$ (the streamwise axis centered between the two rows) and a suitable $x$ translation, and similarly with an additional reflection about $x=0$ (the vertical axis of one of the vortices). In both cases, it is sufficient to satisfy (2.2) along the boundary of a vortex in either row. For vortices of streamwise symmetry, these two cases are equivalent.

The second choice was picked, giving in place of (2.2)

$$
u+i v=\frac{\Gamma}{2 \pi A}\left\{\int_{\Sigma_{1}} \log \left|\sin \frac{\pi}{l}\left(z-z^{\prime}\right) \sin \frac{\pi}{l}\left(z+z^{\prime}-\mu l+i \kappa l-2 \bar{z}\right)\right| d z^{\prime}\right\}+U_{s},
$$

where $\bar{z}$ denotes the centroid of $\Sigma_{1}$ and is for now assumed to be arbitrary. The first symmetry could also have been considered, but since only streamwise symmetric solutions were found using (2.3), this was not attempted.

Three quantities of interest are the propagation velocity $U_{s}$ of the array, the kinetic energy of the fluid, and the momentum transport. They are needed to relate this model to the wake flow problem. Specifically, the following quantities are defined:

$$
T=\frac{1}{2 l} \int_{-\infty}^{+\infty} \int_{-l / 2}^{l / 2}\left(u^{\prime 2}+v^{2}\right) d x d y, \quad D^{\prime}=-\frac{1}{2} \operatorname{Im} \int_{-i \infty}^{+i \infty}\left(u^{\prime}-i v\right)^{2} d z,
$$

where the contour integral in the expression for $D^{\prime}$ is along any contour from $y=-\infty$ to $y=+\infty$ which does not pass through a vortex. Here $T$ is the kinetic energy of the fluid per unit length (streamwise), $D^{\prime}$ is essentially the momentum flux of the fluid in the streamwise direction with the contribution from the vortices themselves omitted, and $u^{\prime}=u-U_{s}$ is the $x$ velocity relative to the free stream. Dimensionless values of these quantities are defined as follows:

$$
\hat{U}_{s}(\alpha, \kappa)=\frac{l}{\Gamma} U_{s}, \quad \hat{T}(\alpha, \kappa)=\frac{l}{\Gamma^{2}} T, \quad \hat{D}^{\prime}(\alpha, \kappa)=\frac{l}{\Gamma^{2}} D^{\prime} .
$$

3. Numerical method. Two successful numerical schemes were employed to calculate the steady vortex shapes, one using Newton's method in a straightforward manner and the other using an ad hoc iterative scheme (Pierrehumbert and Widnall [8]). Only solutions for vortices symmetric in the streamwise direction were computed. The first numerical scheme allowed solutions lacking this symmetry, but none were found (although an exhaustive search was not conducted). For purposes of the calculations, $\Sigma_{1}$ was taken to be the vortex with centroid at the origin. 
The condition that the vortex boundary be a streamline may be written:

$$
\operatorname{Im}\left\{\frac{\partial z^{*}}{\partial s}(u+i v)\right\}=0
$$

where the derivative is taken along the boundary. The boundary of $\Sigma_{1}$ is parameterized using polar coordinates:

$$
\begin{array}{ll}
z=R(\tilde{\vartheta}) e^{i \vartheta(\tilde{\vartheta})}, & 0 \leqq \tilde{\vartheta} \leqq 2 \pi, \\
\vartheta(\tilde{\vartheta})=\vartheta_{0}+\tilde{\vartheta}-\delta \sin 2 \tilde{\vartheta}, & 0 \leqq \delta<\frac{1}{2}, \\
R(\tilde{\vartheta})=\frac{1}{2} a_{0}+\sum_{j=1}^{N}\left(a_{j} \cos j \tilde{\vartheta}+b_{j} \sin j \tilde{\vartheta}\right) . &
\end{array}
$$

Here $\vartheta_{0}, \delta$ are parameters which permit limited adjustment of the scaling of $\vartheta$ in regions of high curvature of the boundary, so as to improve the rate of convergence of the Fourier series for $R$.

Equation (3.1) is evaluated at uniformly spaced values of $\tilde{\vartheta}$ :

$$
\tilde{\vartheta}=\tilde{\vartheta}_{j} \equiv \frac{2 \pi j}{2 N+1}, \quad j=0,1,2, \cdots, 2 N .
$$

This gives $2 N+1$ equations for the $2 N+2$ unknowns $a_{0}, \cdots, a_{N}, b_{1}, \cdots, b_{N}, U_{s}$. An additional equation comes from fixing the size of the vortex, e.g.

$$
R(\varphi)=\text { fixed, }
$$

where $\varphi$ is some fixed angle. However, the resulting system is singular, because (3.1) is invariant to a translation of $z$. The specification is completed by fixing the centroid of the vortex at the origin, i.e.

$$
\bar{x}+i \bar{y} \equiv \frac{1}{3 A} \int_{\Sigma_{1}} R^{3} e^{i \vartheta} d \vartheta=0 .
$$

The resulting $2 N+4$ real equations for the $2 N+2$ unknowns are not independent and the problem is handled by using the trick (Chen and Saffman [1]) of solving (3.4) combined with the $2 N+1$ equations which arise from the discretization of

$$
\operatorname{Im}\left\{\frac{\partial z^{*}}{\partial s}(u+i v)\right\}+f_{1}(\tilde{\vartheta}) \bar{x}+f_{2}(\tilde{\vartheta}) \bar{y}=0,
$$

where $f_{1}$ and $f_{2}$ are more or less arbitrary nontrivial functions chosen to ensure that the Jacobian of the system is nonzero for the solutions that satisfy (3.5). The choice of $\tilde{\vartheta}$ and $\tilde{\vartheta}^{2}$ respectively for $f_{1}$ and $f_{2}$ was found to be satisfactory. Also, the $\bar{z}$ appearing in (2.3) was dropped to simplify the equations slightly.

If $\delta=0$ then, as the limiting case of touching vortices (in each row) is approached, the curvature of $R$ with respect to $\vartheta$ becomes large near $\vartheta=0$ and $\vartheta=\pi$, and hence convergence of the series for $R$ becomes slow. For large $\kappa, \vartheta_{0}=0, \delta=0.4999$ were used, which concentrates mesh points in these regions of large curvature and hence smooths out $R$ as a function of $\tilde{\vartheta}$. This procedure works well for roughly $\kappa>0.36$; below this point, the vortices are too irregularly shaped for this simple technique to be useful. To speed up some computations, $\vartheta_{0}=-\pi / 2, \delta=0$ were used, and the vortices were assumed to be streamwise symmetric. In this case, the Fourier series for $R$ contains only the cosine terms, and hence the number of unknowns is reduced. Specifically, (3.6) is evaluated at the mesh points (3.3) for $j=1,2, \cdots, N$, and the 
unknowns are $a_{0}, a_{1}, \cdots, a_{N-1}, U_{s}$; the remaining Fourier coefficients are taken to be zero.

Integrals for velocity, centroid, and area were evaluated using the trapezoidal rule, with care taken, in the former case, to preserve formal infinite order accuracy (see appendix A). Initial guesses for Newton's method were provided by using one Euler step to advance from the previous converged solution, starting with small vortices and gradually increasing the size by continuation in the parameter (3.4). However, convergence was observed to be insensitive to the initial guess. Accuracy was ensured by requiring that the highest order Fourier coefficients be sufficiently small, and by checking that increasing $N$ had sufficiently small effect on the results. Values of $N$ from 50 to 400 were found to be adequate for 5-digit precision in the final results. Each iteration required roughly from 1 to 25 seconds using a CDC Cyber 203 computer (64 bit floating point).

The second numerical scheme is essentially a scalar approximation to Newton's method. Consider the variation of the stream-function $\psi$ along the boundary of the vortex at some intermediary stage in the calculation. It is assumed that most of the change in $\psi$ on the boundary due to a perturbation of the boundary comes from the fact that it is computed at a different point, rather than the fact that the flow field is changed. Again, a polar coordinate representation for the boundary is employed:

$$
z(\tilde{\vartheta})=R(\tilde{\vartheta}) e^{i \vartheta(\tilde{\vartheta})}, \quad \vartheta(\tilde{\vartheta})=-\frac{\pi}{2}+\tilde{\vartheta}-\delta \sin 2 \tilde{\vartheta}
$$

but here streamwise symmetry of each vortex is assumed ab initio, and the unknowns are taken to be $U_{s}$ and the values of $R$ at

$$
\tilde{\vartheta}=\tilde{\vartheta}_{j} \equiv \frac{\pi j}{N}, \quad j=0,1,2, \cdots, N
$$

The iteration performed can be written

$$
R_{j}^{(n+1)}=R_{j}^{(n)}-\rho \frac{\psi_{j}^{(n)}-\psi_{0}^{(n)}}{\psi_{R, j}^{(n)}}, \quad j=1,2, \cdots, N .
$$

The numerator of the quotient is obtained by integration of the velocity using the trapezoidal rule:

$$
\begin{aligned}
& \psi_{j}^{(n)}-\psi_{j-1}^{(n)} \\
& \approx \frac{\pi}{2 N}\left\{\left(\frac{\partial \psi}{\partial R}\right)_{j}^{(n)}\left(\frac{\partial R}{\partial \tilde{\vartheta}}\right)_{j}^{(n)}+\left(\frac{\partial \psi}{\partial \vartheta}\right)_{j}^{(n)}\left(\frac{\partial \vartheta}{\partial \tilde{\vartheta}}\right)_{j}^{(n)}+\left(\frac{\partial \psi}{\partial R}\right)_{j-1}^{(n)}\left(\frac{\partial R}{\partial \tilde{\vartheta}}\right)_{j-1}^{(n)}+\left(\frac{\partial \psi}{\partial \vartheta}\right)_{j-1}^{(n)}\left(\frac{\partial \vartheta}{\partial \tilde{\vartheta}}\right)_{j-1}^{(n)}\right\},
\end{aligned}
$$

and $\psi_{R} \equiv \partial \psi / \partial R$ is of course a velocity component. The relaxation factor $\rho$ is adjusted empirically for optimum convergence (typically $0.5 \leqq \rho \leqq 2$ ). Cycles of this iteration alternate with an update of $U_{s}$ :

$$
U_{s}^{(n+1)}=U_{s}^{(n)}-\rho \frac{\nabla \psi^{(n)}}{y_{N}-y_{0}}
$$

where $\nabla \psi^{(n)}$ is obtained by integrating $d \psi$ around the half revolution from $\tilde{\vartheta}_{0}$ to $\tilde{\vartheta}_{N}$. The velocity was calculated essentially as before (but see appendix A). As the final step of the iteration, the vortex is shifted in the $y$ direction to put the centroid at the origin, new values of $\boldsymbol{R}_{\boldsymbol{j}}$ being computed via interpolation. 
This method has apparent advantages over Newton's method, namely, its simplicity and its speed per iteration (the cost is $O\left(N^{2}\right)$ per iteration versus $O\left(N^{3}\right)$ for Newton's method). However, highly unpredictable dependence on the initial guess and poor convergence rate in some cases is the penalty. Convergence is geometric with observed convergence factor ranging from about 0.15 for very small vortices to about 0.85 for large vortices. The method failed entirely to converge for very large vortices with small $\kappa$. Furthermore, more points are required for the same accuracy as compared with the previous method. Instability is controlled by limiting the maximum value of $\rho$ and was not a difficulty. Values of $N$ ranging from 50 to 400 were found to be adequate. These calculations were performed using a DEC VAX11/780 computer (64 bit floating point).

The energy can be obtained by a single contour integral over the vortex boundary requiring $O(N)$ operations (see appendix B). The momentum integral was computed by applying the trapezoidal rule over a finite contour passing between two neighboring vortices, and extending to regions where the flow is essentially a uniform stream (the perturbation decays exponentially with $y$ ). Romberg integration was used to obtain sufficient accuracy for this calculation.

4. Circular vortex approximation. The vortices of small area for the exact problem are nearly circular, and for precisely circular vortices the propagation velocity, momentum transport and energy calculations can be done analytically. The former two calculations lead to the same result as for point vortices (Goldstein [3]), since the flow field outside a uniform circular vortex is identical to that of a point vortex of the same circulation. For $d=l / 2, \mu=\frac{1}{2}$,

$$
U_{s}=\frac{\Gamma}{2 l} \tanh \frac{\pi h}{l}, \quad D^{\prime}=\frac{\Gamma^{2}}{2 \pi l}-\frac{\Gamma h}{l} U_{s},
$$

and the energy can be evaluated exactly by integration of the kinetic energy density:

$$
T=\frac{\Gamma^{2}}{2 \pi l}\left[\log \left\{\left(\frac{l^{2}}{\pi A}\right)^{1 / 2} \cosh \frac{\pi h}{l}\right\}+\frac{1}{4}\right] .
$$

Note that the circular vortex model loses physical validity when the vortices overlap, namely for $\alpha>\min \left\{(\pi / 4),(\pi / 4)\left(\kappa^{2}+\frac{1}{4}\right)\right\}$.

5. Results of the calculations. Figures $2-5$ show the calculated values of $\hat{U}_{s}, \hat{T}$, and $\hat{D}^{\prime}$ for the exact problem, accompanied by the corresponding results for the circular vortex approximation. The curves were traced by using as continuation parameter the quantity $a$ which is the ratio of the $x$ semi-axis of the vortices to $l$. As is evident in Fig. 5, a solution of simultaneous maximum area and minimum energy exists for each $\kappa$ in accordance with Kelvin's variational principle for the steady states (Saffman and Szeto [10]). This limit is a contour in the $(\kappa, \alpha)$ plane, as depicted by curve 1 in Fig. 6 . For roughly $\kappa>0.36$, further increase in $a$ results in a decrease in area and increase in energy, up to the point $a=0.5$, where the vortices in each row touch. This limiting case is depicted by curve 2 in Fig. 6 . Thus, between curves 1 and 2 there are two different configurations for a given $(\kappa, \alpha)$. Presumably, the solution curves could be continued beyond $a=0.5$ by considering two adjacent distorted vortex layers in place of discrete vortices, but this was not done. Similar behavior has been observed for the linear vortex array, which in fact corresponds to the limit $\kappa \rightarrow \infty$ (Saffman and Szeto [10]). Quite different behavior was observed for $\kappa$ smaller than about 0.36 . In this case, the calculations indicate that the parameter $a$ approaches a limiting value less than 0.5 . To 

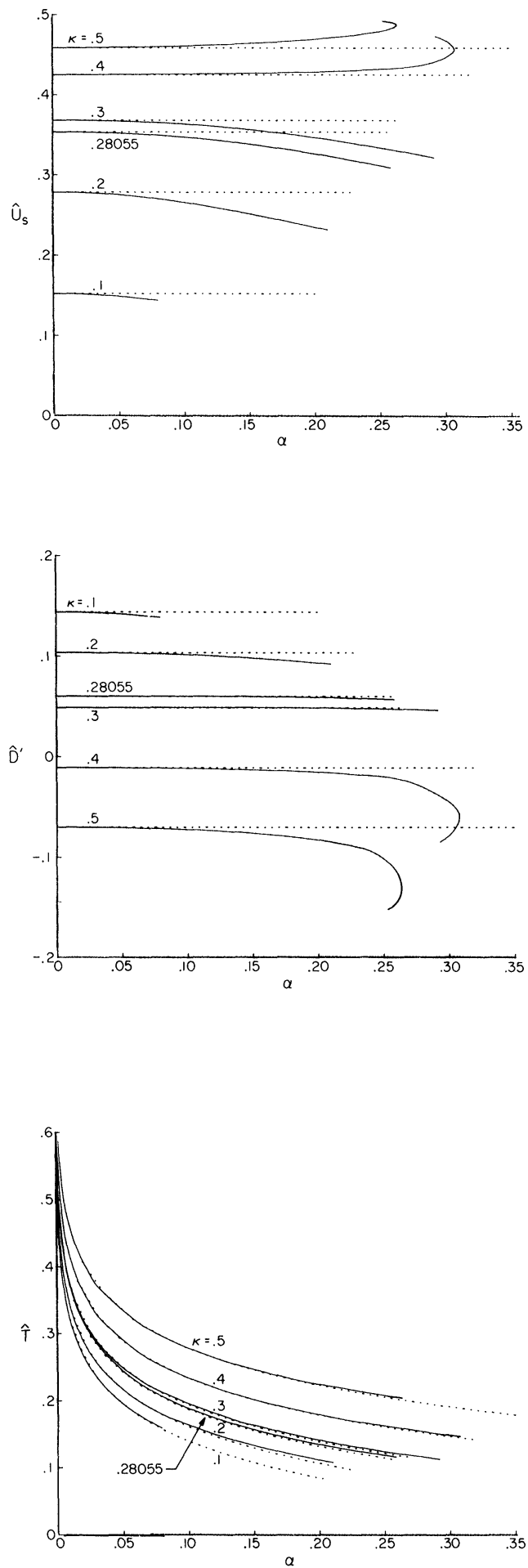

Figs. 2-4. Values of $\hat{U}_{s}, \hat{D}^{\prime}$ and $\hat{T}$ for the fully infinite vortex array. Solid lines denote the calculated values for the exact problem and dashed lines denote the circular vortex approximation. 


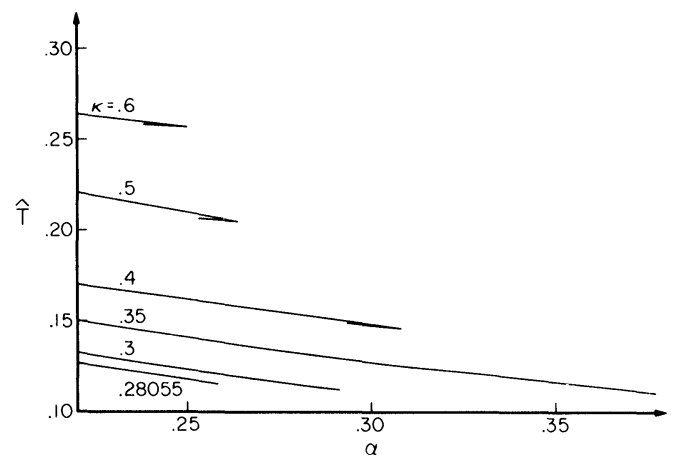

FIG. 5. Expanded plot of energy $\hat{T}$ versus area $\alpha$, showing the nonuniqueness, maximum area and minimum energy.

check that this phenomenon was not dependent on the choice of the horizontal semi-axis for continuation, the vertical semi-axes were also used as continuation parameters. In all cases, the numerical evidence indicates that as the vortex size increases, vortices in each row protrude between vortices in the other row, and the solutions branches terminate when vortices in opposite rows approach and finally meet. Here there is no turnaround in area or energy, but maximum area and minimum energy occur at the limiting point of the solution branch. This behavior is similar to that observed for a pair of counter-rotating vortices as studied by Pierrehumbert [7]. The calculations for $\kappa<0.36$ and for large area were costly, and an accurate calculation of the limiting case was not attempted. For this region, the corresponding segment of curve 1 in Fig. 6 should be regarded as a lower bound.

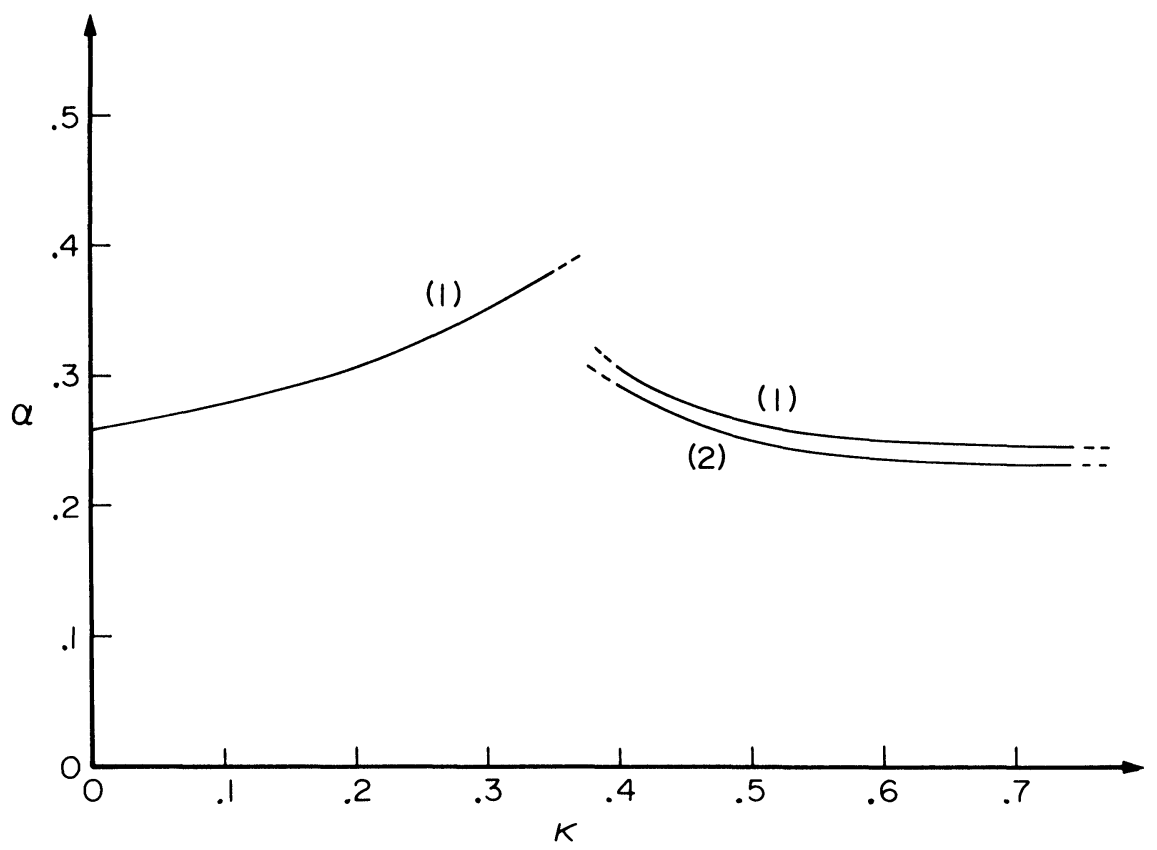

FIG. 6. A plot of the area $\alpha$ versus spacing ratio $\kappa$ plane. Curve 1 denotes the maximum area for a given spacing ratio. The segment corresponding to smaller $\kappa$ should be regarded as a lower bound. Above curve 2 , there are two solutions for a given pair $(\alpha, \kappa)$. 
Presumably, there exists a critical value of $\kappa$ which divides the regions of the two types of limiting behavior. Due to cost limitations, it was not possible to determine accurately this critical value. However, it is believed to lie within the range from 0.35 to 0.365 .

A geometric observation of relevance is that for small areas and for $\kappa>0.36485$, the vortices are longer in the streamwise direction than in the transverse, and the converse for $\kappa$ less than this critical value. The exact dividing value for infinitesimal area is the solution to $\cosh ^{2} \pi \kappa=3$, which may be demonstrated using an elliptical vortex approximation (as has been applied to the linear vortex array; see Saffman and Szeto [10]). This is in good agreement with the numerically estimated large area critical value of $\kappa$ as discussed above, but there is no evidence to suggest that the large area critical value is precisely the infinitesimal area critical value.

Figures 7-13 are plots showing the vortex shapes and the velocity fields. The apparent good qualitative agreement with experimental observations (for example,

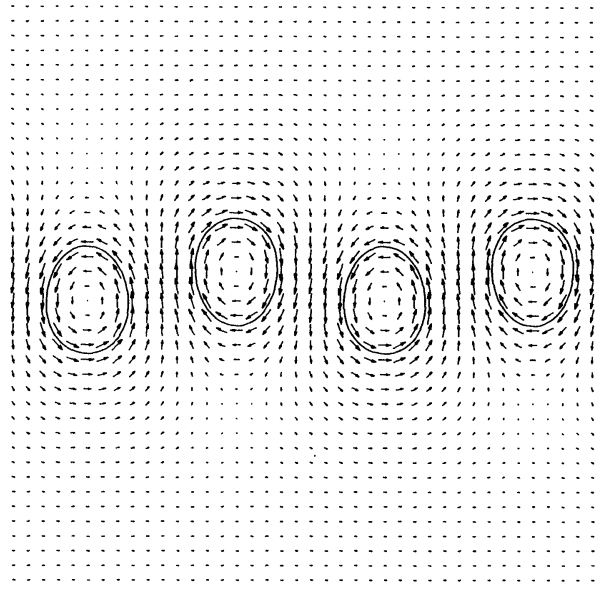

FIG. 7. $\kappa=0.1, \alpha=0.07948$.

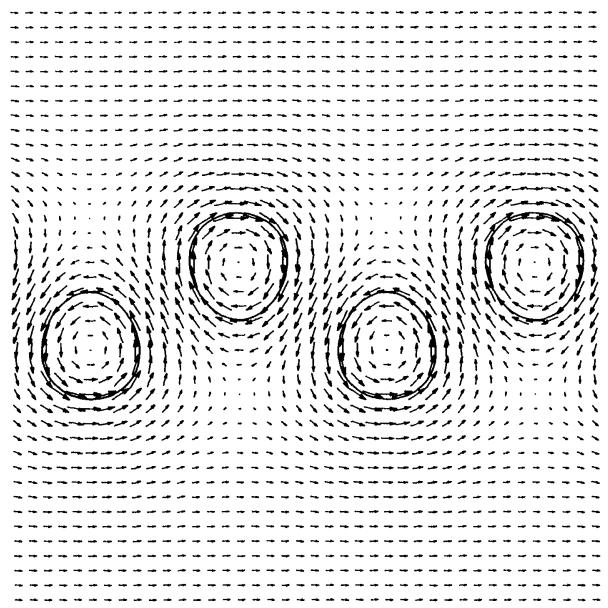

FIG. 9. $\kappa=0.28055, \alpha=0.09382$.

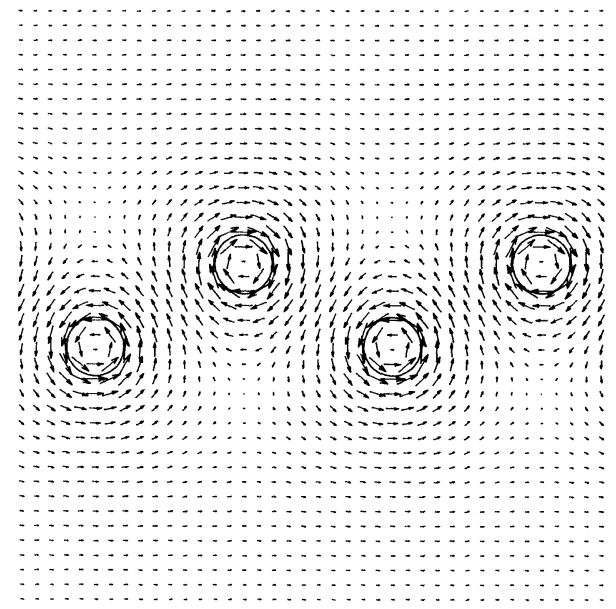

FIG. 8. $\kappa=0.28055, \alpha=0.03011$.

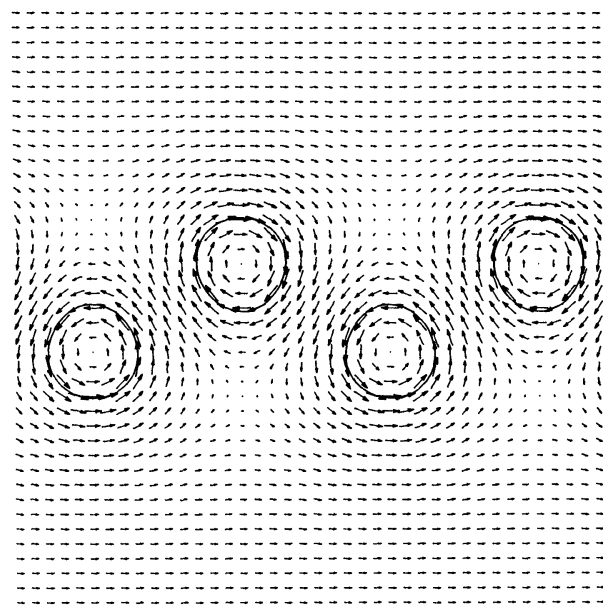

FIG. 10. $\kappa=0.3, \alpha=0.07484$. 


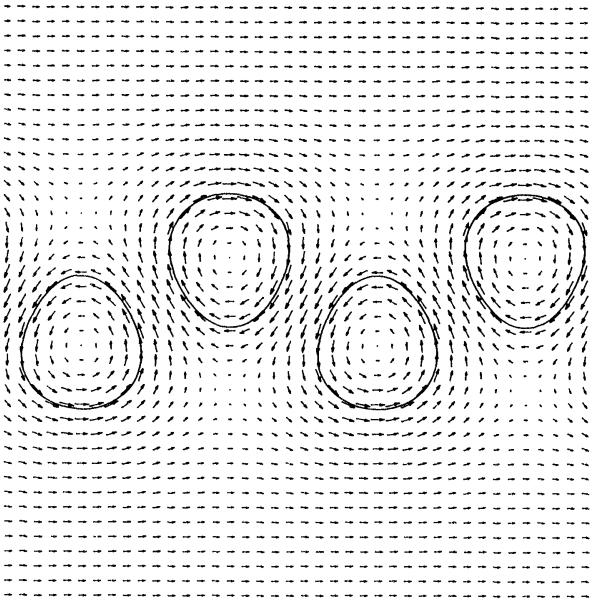

FIG. 11. $\kappa=0.3, \alpha=0.1409$.

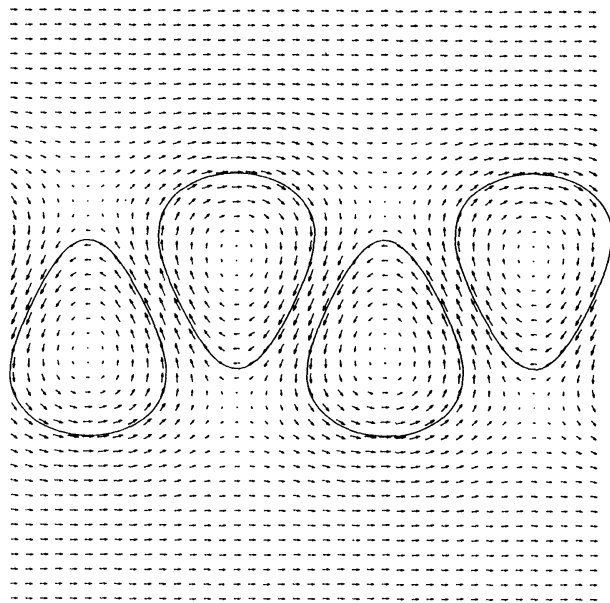

FIG. 12. $\kappa=0.3, \alpha=0.2541$.

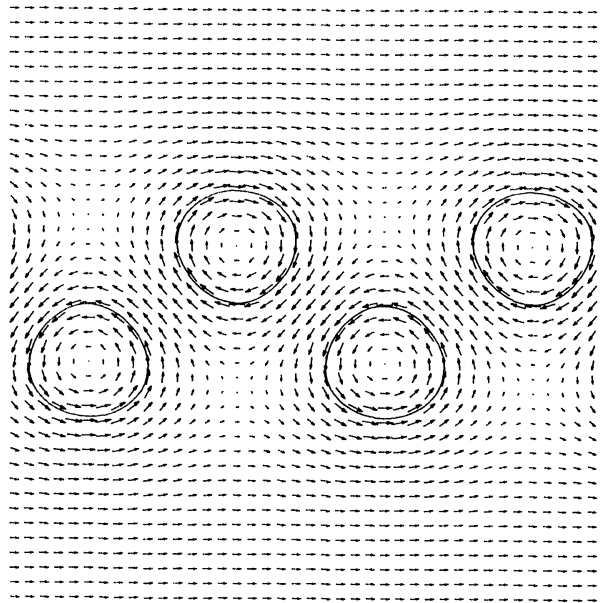

FIG. 13. $\kappa=0.4, \alpha=0.1188$.

FIGS. 7-13. Plots of the vortex shapes and velocity field. Arrow length is proportional to the speed of the fluid at midpoint.

Davies [2]) seems to provide some justification for the assumptions implicit in the forthcoming application of this model to the wake behind bluff bodies and its stability.

Appendix A. Calculation of the velocity. To evaluate the integrals in (2.2) it is useful to write

$$
\int_{\Sigma_{1}} \log \left|\sin \frac{\pi}{l}\left(z-z^{\prime}\right)\right| d z^{\prime}=\int_{0}^{2 \pi} \log \left|\sin \frac{\pi}{l}(z-Z)\right| \frac{d Z}{d \Theta} d \Theta
$$

where $\left.Z \equiv z\right|_{\tilde{\vartheta}=\Theta}$ and $d Z / d \Theta$ can be determined from (3.2) as finite Fourier series in $\Theta$. Hence, the integrand is $2 \pi$-periodic, and the trapezoidal rule gives formally infinite order accuracy-provided that the integrand has infinitely many derivatives, i.e., that $z$ 
is not on the boundary of the vortex. Unfortunately, $z$ is on the boundary for one of the integrals when (3.6) is solved to obtain the vortex shapes.

To preserve accuracy in this case, the following trick is used (suggested by Dr. B. Fornberg):

$$
\int \log \left|\sin \frac{\pi}{l}\left(z-z^{\prime}\right)\right| d z^{\prime}=\int \log \left|\frac{\sin \frac{\pi}{l}\left(z-z^{\prime}\right)}{\frac{\pi}{l}\left(z-z^{\prime}\right)}\right| d z^{\prime}+\int \log \left|\frac{\pi}{l}\left(z-z^{\prime}\right)\right| d z^{\prime} .
$$

The first integral presents no difficulties, and the second can be calculated as follows:

$$
\begin{aligned}
\int \log \left|\frac{\pi}{l}\left(z-z^{\prime}\right)\right| d z^{\prime} & =\int \log \frac{\pi}{l}\left(z-z^{\prime}\right) d z^{\prime}-\int \log \frac{z-z^{\prime}}{\left|z-z^{\prime}\right|} d z^{\prime} \\
& =-i \int \arg \left(z-z^{\prime}\right) d z^{\prime} \\
& =-i \int_{\tilde{\vartheta}}^{\tilde{\vartheta}+2 \pi} \arg (z-Z) \frac{d Z}{d \Theta} d \Theta \\
& =-i \int_{\tilde{\vartheta}}^{\tilde{\vartheta}+2 \pi}\left[\arg (z-Z)-\frac{1}{2} \Theta\right] \frac{d Z}{d \Theta} d \Theta-\frac{1}{2} i \int_{\tilde{\vartheta}}^{\tilde{\vartheta}+2 \pi} \Theta \frac{d Z}{d \Theta} d \Theta \\
& =-i \int_{\tilde{\vartheta}}^{\tilde{\vartheta}+2 \pi}\left\{\left[\arg (z-Z)-\frac{1}{2} \Theta\right] \frac{d Z}{d \Theta}-\frac{1}{2} Z\right\} d \Theta-i \pi z(\tilde{\vartheta}),
\end{aligned}
$$

where the arg function is taken so that the integrand is $2 \pi$-periodic.

For the second numerical scheme, $\boldsymbol{R}_{\vartheta}$ was approximated by a fourth order centered finite difference formula, and $z_{\vartheta}$ then obtained from this. Hence, the integrals were approximated to third order.

Appendix B. Energy calculation. The calculation of the kinetic energy for the infinite vortex street proceeds nearly identically as for the infinite linear array (Saffman and Szeto [10]). The result (with unit density) may be written

$$
T=\frac{1}{4} \frac{\Gamma}{A} \int_{\Sigma_{1}} R^{2} \frac{\partial \psi}{\partial n} d s-\frac{\Gamma}{2 \pi} \int_{\Sigma_{1}} \chi \frac{\partial \psi}{\partial n} d s-\frac{1}{16} \frac{\Gamma^{2}}{A^{2}} \int_{0}^{2 \pi} R^{4} d \vartheta
$$

Here $(R, \vartheta)$ are polar coordinates with origin at the centroid of the prime vortex, and $\chi$ is the integrand in (2.1) that gives the value of the stream function at the origin, after combining the integrals; that is,

$$
\psi(0,0)=\frac{\Gamma}{2 \pi A} \iint_{\Sigma_{1}} \chi d A .
$$

The functional form of $\chi$ depends on the symmetry presumed to exist between the two rows of the street. The actual function that was used in these calculations is

$$
\chi(z)=\log \left|\frac{\sin \frac{\pi}{l} z}{\sin \frac{\pi}{l}(z-d+i h)}\right| .
$$

Acknowledgments. We acknowledge with gratitude the granting of time by Control Data Corporation on the Cyber 203 computer at the CDC Service Center, Arden Hills, Minnesota. 


\section{REFERENCES}

[1] B. CHEN AND P. G. SAFFMAN, Numerical evidence for the existence of new types of gravity waves of permanent form on deep water, Stud. Appl. Math., 62 (1980), pp. 1-21.

[2] M. E. DAviES, A comparison of the wake structure of a stationary and oscillating bluff body, using a conditional averaging technique, J. Fluid Mech., 75 (1976), pp. 209-231.

[3] S. Goldstein, ed., Modern Development in Fluid Dynamics, Vol. II, Clarendon Press, Oxford, 1938, pp. 556-565.

[4] T. v. KÁRMÁN, Über den Mechanismus des Widerstands, den ein bewegter Körper in einer Flüssigkeit erfährt, Göttinger Nachr. Math. Phys. K1.,(1911), pp. 509-517.

[5] — Über den Mechanismus des Widerstands, den ein bewegter Körper in einer Flüssigkeit erfährt, Göttinger Nachr. Math. Phys. Kl., (1912), pp. 547-556.

[6] T. v. KÁRMÁN AND H. L. RuBACH, Über den Mechanismus des Flüssigkeits- und Luftwiderstands, Phys. Z., 13 (1912), pp. 49-59.

[7] R. T. Pierrehumbert, $\boldsymbol{A}$ family of steady, translating vortex pairs with distributed vorticity, J. Fluid Mech., 99 (1980), pp. 129-144.

[8] R. T. PIERREHUMBERT AND S. E. WIDNALL, The structure of organized vortices in a shear layer, AIAA Paper 79-1560, 1979.

[9] L. ROSENHEAD, Double row of vortices with arbitrary stagger, Proc. Cambridge Philos. Soc., 25 (1929), pp. 132-138.

[10] P. G. SAFFMAN AND R. Szeto, Structure of a linear array of uniform vortices, to appear, 1981. 